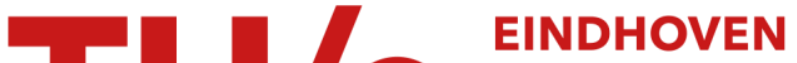 UNIVERSITY OF TECHNOLOGY
}

\section{Amorphization of Si(100) by Ar+ -ion bombardment studied with spectroscopic and time-resolved second-harmonic generation}

\section{Citation for published version (APA):}

Gevers, P. M., Gielis, J. J. H., Beijerinck, H. C. W., Sanden, van de, M. C. M., \& Kessels, W. M. M. (2010). Amorphization of $\mathrm{Si}(100)$ by $\mathrm{Ar}+$-ion bombardment studied with spectroscopic and time-resolved secondharmonic generation. Journal of Vacuum Science and Technology A: Vacuum, Surfaces, and Films, 28(2), 293301. https://doi.org/10.1116/1.3305812

DOI:

10.1116/1.3305812

Document status and date:

Published: 01/01/2010

\section{Document Version:}

Publisher's PDF, also known as Version of Record (includes final page, issue and volume numbers)

\section{Please check the document version of this publication:}

- A submitted manuscript is the version of the article upon submission and before peer-review. There can be important differences between the submitted version and the official published version of record. People interested in the research are advised to contact the author for the final version of the publication, or visit the DOI to the publisher's website.

- The final author version and the galley proof are versions of the publication after peer review.

- The final published version features the final layout of the paper including the volume, issue and page numbers.

Link to publication

\footnotetext{
General rights

- You may freely distribute the URL identifying the publication in the public portal. follow below link for the End User Agreement:

www.tue.nl/taverne

\section{Take down policy}

If you believe that this document breaches copyright please contact us at:

openaccess@tue.nl

providing details and we will investigate your claim.
}

Copyright and moral rights for the publications made accessible in the public portal are retained by the authors and/or other copyright owners and it is a condition of accessing publications that users recognise and abide by the legal requirements associated with these rights.

- Users may download and print one copy of any publication from the public portal for the purpose of private study or research.

- You may not further distribute the material or use it for any profit-making activity or commercial gain

If the publication is distributed under the terms of Article $25 \mathrm{fa}$ of the Dutch Copyright Act, indicated by the "Taverne" license above, please 


\title{
Amorphization of $\mathrm{Si}(100)$ by $\mathrm{Ar}^{+}$-ion bombardment studied with spectroscopic and time-resolved second-harmonic generation
}

\author{
P. M. Gevers, J. J. H. Gielis, H. C. W. Beijerinck, \\ M. C. M. van de Sanden, and W. M. M. Kessels ${ }^{a}$ ) \\ Department of Applied Physics, Eindhoven University of Technology, P.O. Box 513, 5600 MB Eindhoven, \\ The Netherlands
}

(Received 12 October 2009; accepted 11 January 2010; published 5 February 2010)

\begin{abstract}
The surface and interface sensitive technique of optical second-harmonic generation (SHG) has been applied spectroscopically and time-resolved before, during, and after low energy (70-1000 eV) $\mathrm{Ar}^{+}$-ion bombardment of $\mathrm{H}$-terminated $\mathrm{Si}(100)$. The photon energy range of the fundamental radiation was $\hbar \omega=0.76-1.14 \mathrm{eV}$. Besides physical sputtering of the silicon, ion bombardment of crystalline silicon damages and amorphizes the top layer of the sample and thereby creates a layered structure of amorphous silicon $(a-\mathrm{Si})$ on crystalline silicon. The SHG radiation, which is sensitive to the $\mathrm{Ar}^{+}$-ion flux, ion energy, and the presence of reactive gas species, originates from the top surface of the sample and from the interface between $a-\mathrm{Si}$ and $c$-Si. From a comparison with the SHG results obtained at a fundamental radiation of $\hbar \omega=1.3-1.7 \mathrm{eV}$, it is concluded that the SHG radiation during and after creation of this structure dominantly originates from the tails of electronic transitions in the $E_{0}^{\prime} / E_{1}$ energy region rather than from silicon dangling bonds. (C) 2010 American Vacuum Society. [DOI: 10.1116/1.3305812]
\end{abstract}

\section{INTRODUCTION}

The miniaturization of device structures is a key economical driver for the semiconductor industry. To maintain the required progress in the manufacturing processes, one of the challenges is the need to adequately model the dynamics of damage, amorphization, defect creation, and stress during materials processing, as identified by the International Technology Roadmap for Semiconductors. ${ }^{1}$ A main cause for such issues is the collision cascade resulting from energetic ion bombardment in the dry etching or ion implantation processes used in the semiconductor industry. In conjunction with the modeling, there is the need for experimental data to complement and verify the modeling efforts. Additionally, the complexity of the processing tools and systems require increased insight into the surface reaction mechanisms during processing. ${ }^{2}$ Furthermore, as the surface-to-volume ratio increases the influence of surface defects becomes more important for device performance. In addressing these issues by experiments, in situ diagnostics are favored that are capable of capturing the dynamics of the processes involved during processing, preferably being surface and interface specific.

The use of the nonlinear optical technique of (spectroscopic) second-harmonic generation ( $\mathrm{SHG}$ ) as a diagnostic tool is very interesting, especially for Si-based materials processing. The process of SHG, in which two photons of equal energy are converted into one photon with the sum energy, is intrinsically surface and interface sensitive in centrosymmetric materials such as $c$-Si. Being all-optical, SHG is also noninvasive and suitable for in situ application. These aspects combined with the fact that SHG is sensitive to $\mathrm{Si}$ dangling bonds ${ }^{3-5}$ and Si-Si strained bonds ${ }^{6,7}$ have led to a

a) Author to whom correspondence should be addressed; electronic mail: w.m.m.kessels@tue.nl wealth of studies on $c$-Si surfaces and the technologically important interface of $c-\mathrm{Si} / \mathrm{SiO}_{2}$ (see Ref. 8 and references therein.) The use of SHG in studies related to materials processing with ion bombardment is, however, still limited. Heinz et al. ${ }^{3}$ used $\mathrm{Ar}^{+}$-ion bombardment to disturb the signal of a reconstructed silicon surface, measured at a single photon energy of $\hbar \omega=1.17 \mathrm{eV}$. Gielis et al. ${ }^{9}$ performed a spectroscopic SHG study on $c$-Si during ion bombardment; a study that forms a basis for the work described in this article. Furthermore, related studies, during film growth of $a-\mathrm{Si}: \mathrm{H}$ on $c$-Si, were carried out by Kessels and co-workers. ${ }^{10,11}$ The papers of Gielis and co-workers suggest that it might be possible to measure strained and dangling bonds during materials processing. Because the SHG response from damaged $\mathrm{Si}$ is partially related to amorphous silicon $(a-\mathrm{Si})$, it is also worth mentioning here that several studies have measured the SHG response from $a-\mathrm{Si}_{1}{ }^{12,13}$ hydrogenated $a-\mathrm{Si}$ $(a-\mathrm{Si}: \mathrm{H}),{ }^{14}$ and amorphous silicon nitride $\left(a-\mathrm{SiN}_{x}: \mathrm{H}\right) .{ }^{15}$ With respect to spontaneous etching of $\mathrm{Si}$, as opposed to physically etching $\mathrm{Si}$ by energetic ions, the studies of Haraichi and co-workers ${ }^{16-18}$ are relevant. These studies involved several SHG experiments in which $\mathrm{Si}(111)$ was exposed to small amounts of $\mathrm{Cl}_{2}$ and (laser induced) surface changes were observed.

The interaction of laser radiation with matter, as taking place in SHG experiments, is described by the induced polarization $\mathbf{P}(\omega)=\varepsilon_{0} \chi(\omega) \mathbf{E}(\omega)$ in the material, where $\chi$ is the electric susceptibility of the material and $\mathbf{E}(\omega)$ is the electric field associated with the incoming laser radiation. ${ }^{19}$ When the field intensities are sufficiently high, nonlinear effects have to be included in the description. Within the dipole approximation, the first additional contribution to the linear polarization can be described by ${ }^{3}$ 


$$
\mathbf{P}^{(2)}(2 \omega)=\varepsilon_{0} \overleftarrow{\chi}^{(2)}(2 \omega): \mathbf{E}(\omega) \mathbf{E}(\omega),
$$

where $\overleftrightarrow{\chi}^{(2)}$ is the second-order susceptibility of the material. Symmetry rules dictate that $\chi^{(2)} \equiv 0 \mathrm{~m} / \mathrm{V}$ in the bulk of centrosymmetric materials such as $c$-Si and $a$-Si. At surfaces and interfaces the symmetry is broken and SHG radiation can be generated, thereby allowing SHG to be surface and interface sensitive. However, higher order bulk terms, e.g., quadrupole terms, can also radiate at $2 \omega$ and, in general, cannot be disregarded.

Similar to other optical techniques, SHG is resonantly enhanced by electronic transitions in the sample and can, in principle, yield information about the electronic structure. The experimental signal at a single photon energy can be the superposition of multiple resonances, which can also have different spatial origins. Using the theory reported by Erley et al. ${ }^{20}$ the spectroscopic SHG data of the layered structures can be decomposed into a number of separate resonant contributions. In their approach the spectroscopic SHG intensity I follows ${ }^{9,20}$

$$
I(2 \omega)=\left|\sum_{L} A_{L}(\omega) \chi_{L}^{(2)}(2 \omega)\right|^{2} I_{\text {in }}^{2}(\omega),
$$

where $I_{\text {in }}$ is the intensity of the incident fundamental radiation. The subscript $L$ in this equation refers to the spatial origin (e.g., surface or interface) of the resonances. The factor $A_{L}(\omega)$ denotes complex functions that describe the linear propagation of both the fundamental and the SHG radiation through the system and include linear optical phenomena such as reflection, refraction, and absorption. The surface or interface second-order susceptibility is approximated by a coherent superposition of critical-point-like resonances with excitonic line shapes,

$$
\chi_{L}^{(2)}(2 \omega) \propto \sum \frac{h_{q} e^{i \varphi_{q}}}{2 \omega-\omega_{q}+i \Gamma_{q}},
$$

where $h_{q}$ denotes the (real) amplitude, $\omega_{q}$ is the frequency, $\Gamma_{q}$ is the linewidth, and $\varphi_{q}$ is the excitonic phase of resonance $q$. The line shape is derived from the well-studied linear dielectric function $\varepsilon$ of bulk $c$-Si, where the excitonic line shape represents the best approximation for the $E_{1}$ critical point (CP) ${ }^{21}$ This approximation has proven to be useful in SHG for other transitions than the $E_{1} \mathrm{CP}$ as well. ${ }^{7,9,12,20,22}$ For the approximation in Eq. (3), it is assumed that the SHG response is resonant at $2 \omega$, with the fundamental photon energy far from a resonance. For resonant direct interband transitions at the fundamental photon energy, the $2 \omega$ in the denominator of Eq. (3) can be replaced by $\omega . .^{5}$

This article continues with a short review of the relevant results and conclusions from the previous work on the experimental setup used in this work, as well as from reports in literature (Sec. II). In Sec. III the vacuum and laser setup and experimental procedures are discussed, followed by the results and discussion of spectroscopic and time-resolved SHG measurements in Sec. IV. Finally, some concluding remarks are given in Sec. V.

\section{PREVIOUS STUDIES ON THE $a$-SI/c-SI SYSTEM: STATE OF THE ART}

Previous studies on $\mathrm{Si}(100)$ performed by Stevens et al., ${ }^{23}$ who used spectroscopic ellipsometry (SE), as well as by others, ${ }^{24}$ have shown that ion bombardment of crystalline samples creates a layered system. The ions damage the top of the sample essentially as deep as the penetration depth of the ions, rendering the top layer of the sample amorphous. ${ }^{25}$ The linear optical properties of the amorphized region, the ioninduced $a-\mathrm{Si}$, have been studied and appear to be basically independent of the ion energy and are similar to $a$-Si measured by others in different contexts. ${ }^{26-28}$ Additionally, ion bombardment tends to result in a smooth surface of the sample, although the final value of the (low) roughness is a function of the ion energy. This is in contrast to spontaneous etching of $\mathrm{Si}$ by $\mathrm{XeF}_{2}$, which results in increased surface roughness with increasing $\mathrm{XeF}_{2}$ dose. Time-resolved ion bombardment studies have shown that the initial amorphization of the top layer might be governed by two processes. For example, the evolution of the $a$-Si layer thickness created by $70 \mathrm{eV} \mathrm{Ar}^{+}$ions can be described with 1/e time constants, expressed in $\mathrm{Ar}^{+}$-ion dose of 2 and $9 \mathrm{ML},{ }^{29}$ respectively. Once ion bombardment is terminated the $a-\mathrm{Si}$ layer exhibits bulk relaxation behavior. Stevens et al. ${ }^{23}$ showed that the changes in the linear optical response of the $a-\mathrm{Si} / c-\mathrm{Si}$ system as a result of the relaxation can be described by fitting the apparent change in layer thickness $\Delta d$ using a stretched exponential

$$
\Delta d(t)=\Delta d_{\infty}\left\{1-\exp \left[-(t / \tau)^{\alpha}\right]\right\},
$$

with $\tau=360 \mathrm{~s}, \alpha=0.32$, and $\Delta d_{\infty}$ is the final apparent change in the layer thickness, which is typically of the order of $10 \%$ of the total thickness. Such a stretched exponential decay is commonly used and observed in defect dominated relaxation in amorphous materials, with similar values for $\tau$ and $\alpha{ }^{30,31}$

Molecular dynamics (MD) simulations performed by Humbird et $a l . .^{32}$ substantiate the experimental observations reported by Stevens et $a l^{23}$ The observed time scales, i.e., the dose dependence, of the amorphization process in the experiments are similar in the simulations using the same experimental condition. Humbird et al. remarked that $50 \%$ of the final amorphous layer thickness is achieved within $1 \mathrm{ML}$ of $\mathrm{Ar}^{+}$-ion dose for energies of $50 \mathrm{eV}$ and higher. Humbird et $a l$. also confirmed the assumption of Stevens et al. and Gielis et $a l .{ }^{9}$ of a locally flat interface between $a$-Si and $c$-Si, relevant in the interpretation and evaluation of both $\mathrm{SE}$ and SHG data.

Recently, the SHG response of H-terminated $\mathrm{Si}(100)$ during $\mathrm{Ar}^{+}$-ion bombardment in the photon energy range of $\hbar \omega=1.33-1.75 \mathrm{eV}$ was reported by our group. ${ }^{9}$ The measurements comprised of the time-resolved SHG response on the onset of $\mathrm{Ar}^{+}$-ion bombardment and the spectroscopic response as a function of ion energy. The optical response could be modeled by using the excitonic line shape model discussed in the Introduction with two resonances, while describing the optical system with three components, i.e., vacuum, $a$-Si film, and $c$-Si substrate. It was concluded that 

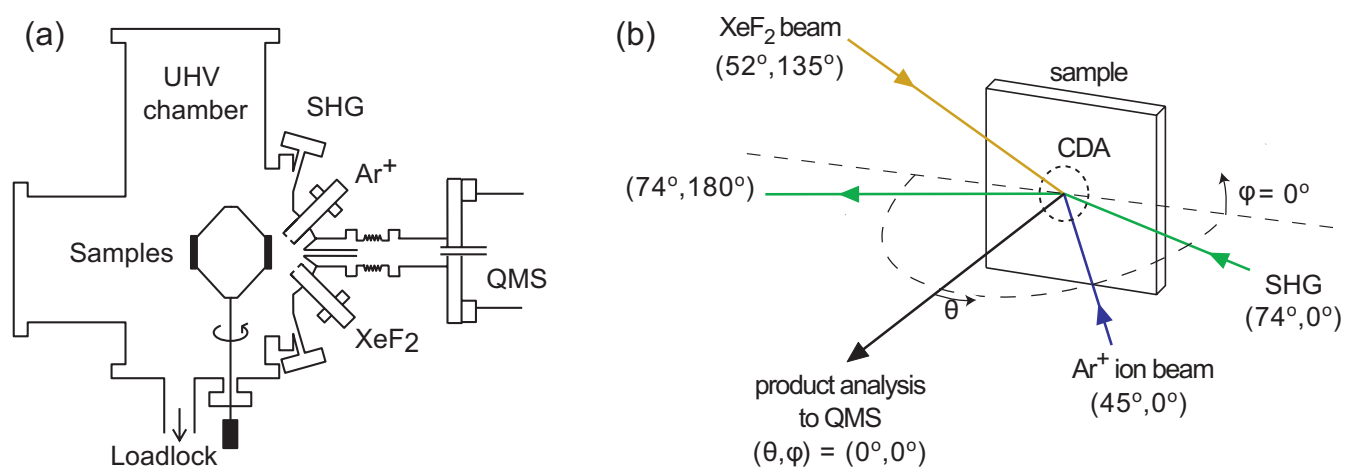

FIG. 1. (Color online) Experimental setup in the horizontal cross section. The sample is rotated in place when loaded and faces the $\mathrm{Ar}^{+}$-ion beam, $\mathrm{XeF}_{2}$ beam, SHG laser beam, and QMS. The spherical coordinates $(\theta, \varphi)$ of the beams are depicted in (b). The central detection area (CDA), which is defined by the QMS, is also depicted.

the SHG response of the samples in this photon energy range is dominated by a two-photon resonance around $2 \hbar \omega$ $=3.36 \mathrm{eV}$ related to the $E_{0}^{\prime} / E_{1}$ transitions in $c-\mathrm{Si}$ near the $a-\mathrm{Si} / c$-Si interface, with an additional two-photon contribution around $2 \hbar \omega=3.2 \mathrm{eV}$ related to $\mathrm{Si}-\mathrm{Si}$ bonds at the $a-\mathrm{Si}$ surface. The energy, linewidth, and the excitonic phase of the contributions were basically independent of the $\mathrm{Ar}^{+}$-ion energy. Exposure of the $a-\mathrm{Si} / c-\mathrm{Si}$ sample to $\mathrm{XeF}_{2}$ could quench the surface contribution, which aided the interpretation of the data in terms of the localization of the resonances. These measurements led to the following picture of the $a$-Si/c-Si system: $\mathrm{Ar}^{+}$-ion bombardment creates an $a-\mathrm{Si}$ layer, which acts as an optical layer. The surface SHG derives from distorted $\mathrm{Si}-\mathrm{Si}$ bonds which can be neutralized by exposure to reactive gases, while the interface contributes to the SHG by $\mathrm{Si}-\mathrm{Si}$ bonds in the first $c$-Si layers below the interface. The latter contribution is stable with respect to sample handling.

To gain further insight, the laser system was extended to facilitate SHG experiments in the photon energy range of $\hbar \omega=0.76-1.14 \mathrm{eV}$, which will be the focus of this work. Combining this photon energy range with that of $\hbar \omega$ $=1.33-1.75 \mathrm{eV}$ provides more information due to a larger total photon energy range. Additionally, the lower photon energies have the advantage of negligible absorption of the fundamental and SHG radiation in the silicon, in contrast to higher photon energies. Finally, the fundamental radiation of $\hbar \omega=0.76-1.14 \mathrm{eV}$ overlaps with the energy region associated with dangling bond states on $c$-Si. It therefore possibly allows for the detection of one-photon resonances of dangling bonds. This could potentially enable the monitoring of defect dynamics related to those dangling bond states. However, it is already noted that this approach needs careful interpretation; when there are multiple contributions from multiple spatial origin, it is easy to overinterpret the data.

\section{EXPERIMENT}

\section{A. Multiple beam setup}

The experimental beam setup has been described extensively in previous publications, ${ }^{9,23,33-35}$ and therefore only the most important features will be described here. Briefly, the setup consists of a high vacuum chamber with a base pressure below $10^{-8}$ mbar. Samples of approximately 10 $\times 10 \mathrm{~mm}^{2}$ in size can be loaded in the chamber from a load lock, which can store up to six samples. When loaded, $\mathrm{Ar}^{+}$ ions from a low energy (10-2000 eV) ion beam (Nonsequitur Technologies, customized version of Model LEIG-2) can be directed on the sample at an angle of $45^{\circ}$ from the normal. Typical average fluxes $\Phi_{\mathrm{Ar}^{+}}$are $0.01 \mathrm{ML} / \mathrm{s}$ for ion energies below $70 \mathrm{eV}$ and $0.4 \mathrm{ML} / \mathrm{s}$ for $1000 \mathrm{eV}^{36}$ The ions are created from $99.999 \%$ pure argon gas. Additionally, the setup is equipped with a well characterized thermally controlled $\mathrm{XeF}_{2}$ neutral beam with an average flux $\Phi_{\mathrm{XeF}_{2}}$ adjustable from 0.05 to $2 \mathrm{ML} / \mathrm{s}$. The $\mathrm{XeF}_{2}$ beam has an angle of incidence of $52^{\circ}$ with respect to the sample surface normal. Figure 1 gives a schematic representation of the setup and of the beams with respect to the sample.

\section{B. Laser system and optics}

The laser system used in this work consists of a series of lasers. A Ti:sapphire oscillator [Spectra Physics (SP) Tsunami, $80 \mathrm{MHz}, \sim 90 \mathrm{fs}$, and $1.55 \mathrm{eV}$ ] is pumped by an intracavity frequency doubled $\mathrm{cw} \mathrm{Nd}: \mathrm{YVO}_{4}$ laser (SP Millennia Vsj). The oscillator, which is tuned to $1.55 \mathrm{eV}$, is used to seed a regenerative Ti:sapphire amplifier (SP Spitfire HPR). The amplifier is pumped by an intracavity frequency doubled $Q$-switched Nd:YLF laser (SP Evolution 30). The resulting laser pulse, which now has a repetition rate of $1 \mathrm{kHz}$, is used to pump an optical parametric amplifier (OPA) (Light Conversion TOPAS-C). The OPA is a two-stage amplifier of whitelight continuum, which is generated in a sapphire plate. The white-light beam is overlapped noncollinearly with pump radiation in a beta-barium borate (BBO) crystal. The resulting signal beam is overlapped collinearly with pump radiation in the second BBO crystal. The signal beam from this setup is in the $0.76-1.14 \mathrm{eV}$ photon energy range, has a pulse duration of $\sim 90 \mathrm{fs}$, and typically has $300 \mu \mathrm{J}$ per pulse depending on the photon energy.

A schematic representation of the optical setup for the measurements is depicted in Fig. 2. The power and polarization of the beam were controlled by a variable wave plate [New Focus (NF) 5540] in combination with a Glan- 


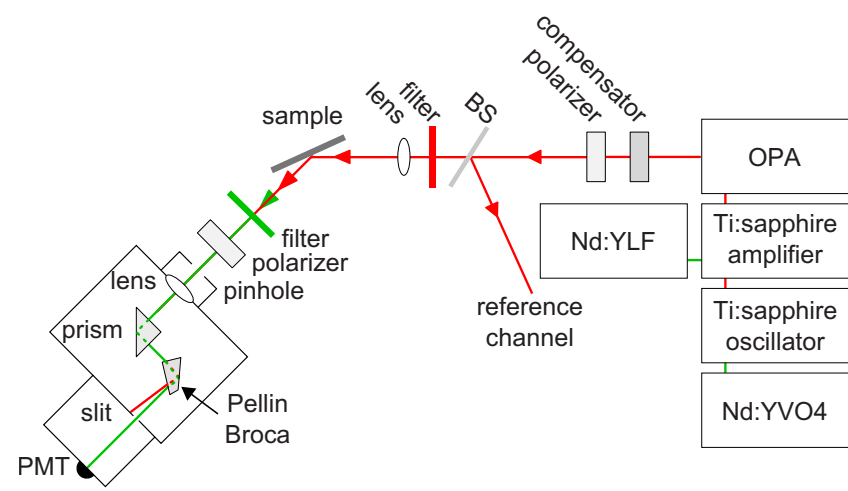

FIG. 2. (Color online) Optical setup to generate and detect SHG in the high vacuum chamber. The reflected beam from the beam splitter follows an optical path containing a reference quartz sample. This optical path with reference sample is equivalent to the depicted path with the sample under study.

Thompson polarizer (NF 5525). All experiments discussed in this article were performed using $p$-polarized fundamental and SHG radiation with an average power of the fundamental radiation around $8 \mathrm{~mW}$. A fraction of the fundamental radiation was split off to provide a reference signal from a single side polished $z$-cut $\alpha$-quartz sample. The remaining part of the beam was directed to the in situ setup. Except for the optical windows of the vacuum setup (stress-free fused silica), both optical paths consisted of similar optical elements. Any SHG present or generated in the optical path was removed by a color filter (Schott RG850) before reaching the sample. The beam was then focused onto the sample using a BK7 lens (CVI PLCX 25.4-103.0-C). In the exit beam the fundamental and third harmonic radiation was suppressed by color filters. Depending on the photon energy, either a combination of Schott KG3 and OG515 filters or a single Schott RG9 was used. The analyzing polarization was set by a polarizer (Thorlabs GL10A for the in situ channel and NF 5524 for the reference channel). The remaining fundamental radiation was spatially separated using a Pellin Broca prism and focused on a slit with a BK7 lens (CVI PLCX 25.4-64.4-C). The SHG radiation was detected using a photomultiplier tube (PMT) (Hamamatsu R928) in combination with an electrical resistor-condensator $(R C)$ circuit. The time dependent response of the voltage on the capacitor of this $R C$ circuit was measured with a $100 \mathrm{MHz}$ transient recorder (TUeDACS), providing a measure for the accumulated charge in the PMT during each laser pulse and, consequently, for the SHG intensity.

\section{Additional diagnostics}

The etch products desorbing from the sample during processing and reflected beam species were monitored with a differentially pumped line-of-sight quadrupole mass spectrometer (QMS) at normal incidence to the sample (see Fig. 1). This QMS is capable of monitoring mass-to-charge ratios $\mathrm{m} / \mathrm{z}$ from 1 to 500 in steps of approximately 0.2 .

Stevens et al. ${ }^{23}$ established the linear optical properties of the samples in an in situ study by using a rotating compen- sator spectroscopic ellipsometer (J.A. Woollam, Inc., $\mathrm{M} 2000 \mathrm{U})$ in the photon energy range of $0.7-5 \mathrm{eV}$. The optical ports used for SE were the same as for the SHG laser beam.

\section{Sample preparation and experiments}

The experiments were performed with $\mathrm{H}$-terminated $\mathrm{Si}(100)$ samples ( $n$-type, phosphorus doped, 10-30 $\Omega \mathrm{cm}$ ). The samples were cleaned and etched by a standard clean (RCA1 and RCA2) with an intermediate and final HF dip (2\%) to remove the native oxide. Immediately after the final rinsing, the samples were transferred into the load lock of the vacuum chamber, preventing reoxidation of the surface. The samples were mounted at an azimuthal angle of $45^{\circ}$ with respect to the [001] crystal axis.

The SHG measurements had a time resolution around 2 ms. Before further processing, the data were normalized point-to-point against the SHG response from the reference quartz sample to reduce shot-to-shot laser beam variations and drift in the laser intensity. Every point in the figures of the time dependent experiments is the average of 1000 data points. Every point in the figures with spectra was averaged over 30 s, i.e., an average over approximately $1.5 \times 10^{4}$ data points.

Because of sample-to-sample fluctuations in the absolute value of the SHG response, mainly due to small variations in the alignment of the sample, the following procedure was used to normalize the results from various experiments. On each sample, after the final measurement, the ion energy and flux were increased to $1000 \mathrm{eV}$ and $0.04 \mathrm{ML} / \mathrm{s}$ respectively, and the SHG response was measured at a photon energy of $\hbar \omega=1.0 \mathrm{eV}$. Because this is the highest ion energy and flux used in the experiments, with the most expected sample damage, the SHG signal in steady state is assumed to be independent of the sample history under $\mathrm{Ar}^{+}$-ion bombardment. All experiments were therefore normalized to that condition. Instead, the data of the experiment with $\mathrm{XeF}_{2}$ dosing were normalized to the steady-state level measured just prior to the actual dosing because it is not investigated yet what the influence of the presence of $\mathrm{F}$ is on the signal during subsequent $\mathrm{Ar}^{+}$-ion bombardment.

To directly compare the spectra obtained in this work with those reported by Gielis et al., ${ }^{9}$ the SHG response of the different experiments was expressed on a common scale. The reflection coefficient ${ }^{37,38} R$, which is independent of the laser beam properties (but not of the angle of incidence), was chosen as a suitable common scale and is defined by

$$
R(2 \omega) \equiv \frac{I(2 \omega)}{\left[I_{\text {in }}(\omega)\right]^{2}}
$$

[compare to Eq. (2)]. The Appendix contains the procedure how the numerical value for $R$ was obtained in this work.

As it is unknown a priori whether the SHG stems from a one-photon, a two-photon transition, or both, the spectroscopic data in this article are presented with both the fundamental (bottom axis) and the second-harmonic photon energy (top axis) depicted in the figures. 


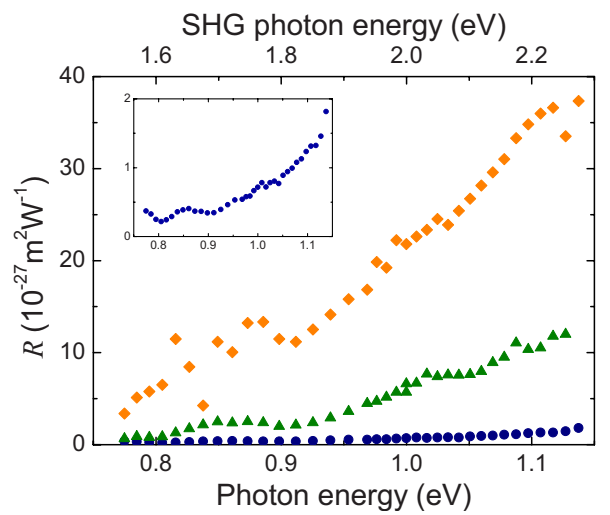

FIG. 3. (Color online) SHG reflection coefficient spectra for $\mathrm{H}-\mathrm{Si}(100)$ (circles), for $c$-Si during $70 \mathrm{eV} \mathrm{Ar}^{+}$-ion bombardment (diamonds), and for $c$-Si which is exposed to a small dose of $\mathrm{XeF}_{2}(\sim 10 \mathrm{ML})$ after $70 \mathrm{eV}$ $\mathrm{Ar}^{+}$-ion bombardment (triangles). In the inset, the $\mathrm{H}-\mathrm{Si}$ spectrum is magnified to show more detail.

\section{RESULTS AND DISCUSSION}

\section{A. Spectroscopic SHG response}

In order to investigate the SHG response from $c$-Si(100) during $\mathrm{Ar}^{+}$-ion bombardment, three SHG reflection coefficient spectra were measured, as shown in Fig. 3. The first spectrum (circles) shows an $\mathrm{H}$-terminated $c$-Si sample (H$\mathrm{Si}$ ), which is the starting condition for the experiments in this article. As can be seen in the inset of Fig. 3, the spectrum contains a broad, not very distinct signature, which increases substantially with increasing photon energy, but apparently without reaching a maximum value for the photon energy range studied. The spectrum contains small scale features, but they are currently believed to be within the experimental error, which is governed by the laser (spatial) pulse shape and the measurement of the average power of the fundamental radiation.

When the sample is exposed to $70 \mathrm{eV} \mathrm{Ar}^{+}$-ion bombardment at a flux of $0.04 \mathrm{ML} / \mathrm{s}$, the SHG signal increases over the whole spectral range by factors of 20-30 (Fig. 3, diamonds). The spectrum is measured under steady-state conditions during the $\mathrm{Ar}^{+}$-ion bombardment, effectively preventing a significant influence on the SHG signal from the presence of background species in the vacuum setup. ${ }^{9}$ The shape of the $\mathrm{H}-\mathrm{Si}$ spectrum and the $70 \mathrm{eV} \mathrm{Ar}^{+}$spectrum is very similar, although the data points at the edges of the spectral range are relatively higher for the $\mathrm{H}-\mathrm{Si}$ spectrum.

In an attempt to separate surface contributions from subsurface contributions, the sample was exposed to a small dose of $\mathrm{XeF}_{2}$. To illustrate the procedure followed, Fig. 4 shows the time line of the experiment from the simultaneously measured QMS signal at a $m / z$ ratio matching $\mathrm{Xe}^{+}$. The $c$-Si sample was exposed to $70 \mathrm{eV} \mathrm{Ar}^{+}$ions from $t$ $=0 \mathrm{~s}$ to $t=600 \mathrm{~s}$. At the moment the $\mathrm{Ar}^{+}$-ion bombardment was stopped, the sample was exposed to the $\mathrm{XeF}_{2}$ beam with a flux of $\sim 1 \mathrm{ML} / \mathrm{s}$ for about $13 \mathrm{~s}$. As the spontaneous etch yield during this phase of dosing is on the order of $0.02 \mathrm{Si}$ per incoming $\mathrm{XeF}_{2},{ }^{34}$ the dose is too small to substantially etch the $a$-Si. The third spectrum in Fig. 3 (triangles) shows

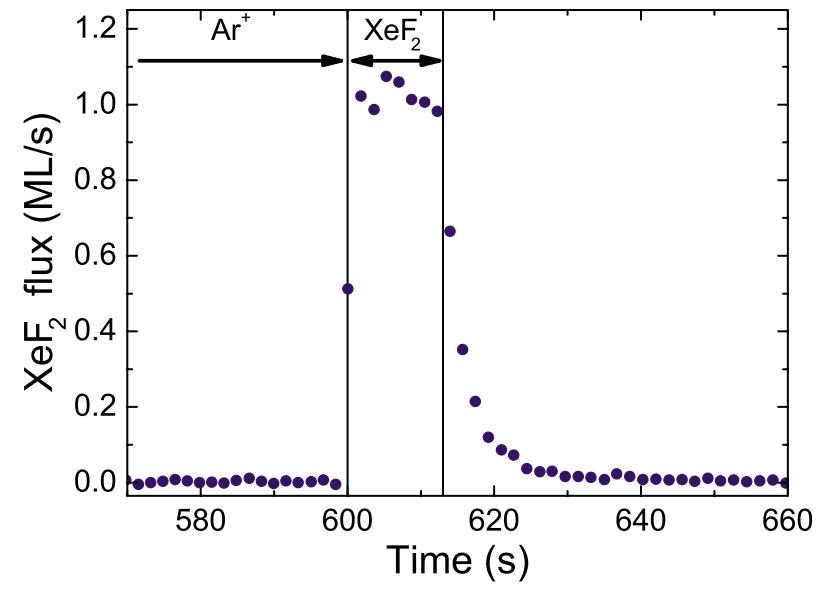

FIG. 4. (Color online) Time dependent QMS signal of $\mathrm{Xe}^{+}$before, during, and after exposure of a $c$-Si sample to $70 \mathrm{eV} \mathrm{Ar}^{+}$ions and $\mathrm{XeF}_{2}$. The exposure to $\mathrm{Ar}^{+}$ions started at $t=0 \mathrm{~s}$ and continued until $t=600 \mathrm{~s}$. The exposure to $\mathrm{XeF}_{2}$ lasted from $t=600 \mathrm{~s}$ to $t=613 \mathrm{~s}$. The steady-state flux of $\mathrm{XeF}_{2}$ at the experimental settings was calibrated to be $1.0 \mathrm{ML} / \mathrm{s}$.

the resulting SHG reflection coefficient. Although the shape of the spectrum remains approximately the same as the spectrum for the sample during $\mathrm{Ar}^{+}$-ion bombardment, the amplitude of the spectrum of the $\mathrm{XeF}_{2}$ dosed sample is approximately three times smaller. This behavior implies that at least a part of the SHG signal during ion bombardment results from the surface of the $a$-Si layer, although it cannot unambiguously be excluded that the change results from an additional surface contribution due to the dosing (a contribution with opposite phase).

Gielis et al. ${ }^{9}$ obtained SHG data in the photon energy range of $\hbar \omega=1.33-1.75 \mathrm{eV}$ on $\mathrm{H}-\mathrm{Si}$ and during $\mathrm{Ar}^{+}$-ion bombardment of $c$-Si, as already discussed in Sec. II. For comparison, their results were converted to $R$ (see Appendix) and are depicted together with the current results in Fig. 5. In addition to the data, Fig. 5 contains three curves resulting from fitting the data in the $\hbar \omega=1.33-1.75 \mathrm{eV}$ photon energy range with excitonic line shapes, in accordance with Eq. (3). The spectrum of $\mathrm{H}-\mathrm{Si}$ is fitted with one two-photon resonance at $\hbar \omega=3.32 \mathrm{eV}$ (continuous line). The fit of the $a$-Si $/ c$-Si stack created by $70 \mathrm{eV} \mathrm{Ar}^{+}$-ion bombardment from Ref. 9 was redone, ${ }^{39}$ and in contrast to the original paper, the new fit was able to converge to acceptable values with all fitting parameters free (dotted line). Additional to the full spectrum, also the spectrum obtained by removing the surface contribution from the model in the $a-\mathrm{Si} / c-\mathrm{Si}$ stack is shown in Fig. 5 (dashed line). The resulting parameters are depicted in Table I.

When the fitted spectra in the photon energy range of $\hbar \omega=1.33-1.75 \mathrm{eV}$ are compared to the measured data in the photon energy range of $\hbar \omega=0.76-1.14 \mathrm{eV}$, the trends suggest that the normalization procedure works well. From the comparison, it can be concluded that the SHG signal at low photon energies can largely be attributed to the tail of the known two-photon resonances in the $E_{0}^{\prime} / E_{1}$ region. This also 


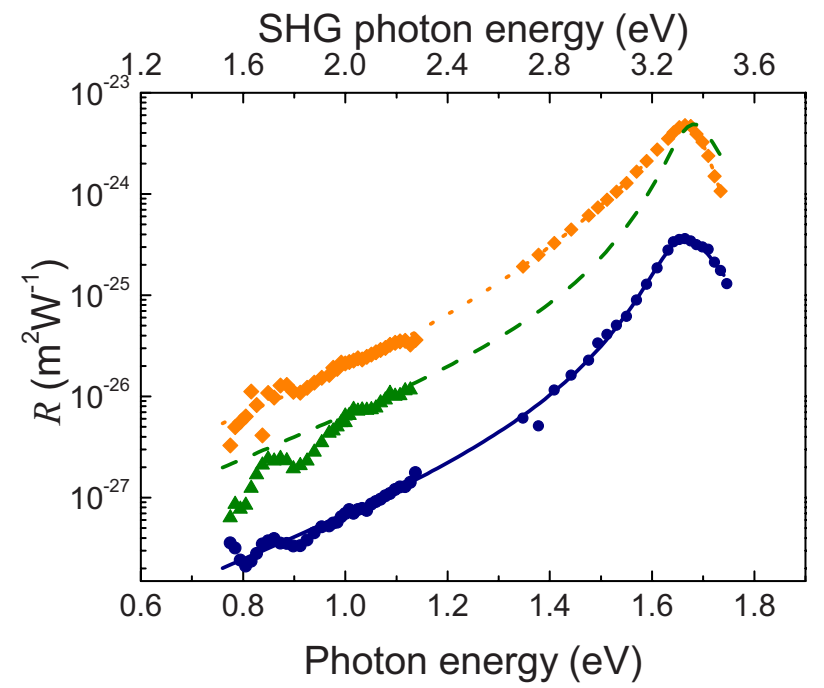

FIG. 5. (Color online) SHG reflection coefficient spectra (on logarithmic scale) for $\mathrm{H}-\mathrm{Si}(100)$ (circles), for $c$-Si during $70 \mathrm{eV} \mathrm{Ar}^{+}$-ion bombardment (diamonds), and for $c$-Si which is exposed to a small dose of $\mathrm{XeF}_{2}$ ( $\sim 10 \mathrm{ML})$ after $70 \mathrm{eV} \mathrm{Ar}^{+}$-ion bombardment (triangles). The data points in the $\hbar \omega=0.76-1.14 \mathrm{eV}$ range are the same as in Fig. 3, the data points in the $\hbar \omega=1.33-1.75 \mathrm{eV}$ range are reproduced from Ref. 9. The lines follow from the model to the data as described in text.

implies that the picture of the layered system with two spatially separated contributions also holds for the $\hbar \omega$ $=0.76-1.14 \mathrm{eV}$ region.

\section{B. Time-resolved SHG response}

To gain insight into the time evolution of the SHG signal, the SHG intensity was monitored at a photon energy of $\hbar \omega$ $=1.0 \mathrm{eV}$ from the start of the $\mathrm{Ar}^{+}$-ion bombardment. All time dependent experiments started with a clean $\mathrm{H}-\mathrm{Si}$ sample, which was subsequently exposed to $\mathrm{Ar}^{+}$ions for 10 min. In Fig. 6, the response for $70 \mathrm{eV} \mathrm{Ar}^{+}$-ion fluxes of 0.01 , 0.02 , and $0.04 \mathrm{ML} / \mathrm{s}$ is depicted. The latter condition is the same as for the spectra discussed in Sec. IV A. The SHG signal for the 0.02 and $0.04 \mathrm{ML} / \mathrm{s}$ measurements showed a steep increase in the first $5 \mathrm{~s}$ of $\mathrm{Ar}^{+}$-ion exposure to approximately $25 \%$ of the final steady-state value, while the 0.01 $\mathrm{ML} / \mathrm{s}$ sample showed a much slower response. After the initial increase the response slowly reached a steady state which was obtained after several ML of $\mathrm{Ar}^{+}$-ion dose. The steadystate value for the $0.04 \mathrm{ML} / \mathrm{s}$ experiment was a factor 60 higher than the starting value of the SHG signal on $\mathrm{H}-\mathrm{Si}$, while this difference was smaller when lower fluxes were

TABLE I. Parameters used for the modeling of the spectra in Fig. 5 using Eqs. (2) and (3).

\begin{tabular}{|c|c|c|c|c|}
\hline Location & $\begin{array}{l}h_{q} \\
\text { (a.u.) }\end{array}$ & $\begin{array}{l}\hbar \omega_{q} \\
(\mathrm{eV})\end{array}$ & $\begin{array}{l}\hbar \Gamma_{q} \\
(\mathrm{eV})\end{array}$ & $\begin{array}{c}\varphi_{q} \\
(\mathrm{rad})\end{array}$ \\
\hline H-Si surface & $2.89 \pm 0.06$ & $3.322 \pm 0.002$ & $0.128 \pm 0.003$ & 0 \\
\hline$a$-Si surface & $14 \pm 2$ & $3.2 \pm 0.1$ & $0.46 \pm 0.07$ & $(1.4 \pm 0.2) \pi$ \\
\hline $\begin{array}{c}a-\mathrm{Si} / c-\mathrm{Si} \\
\text { interface }\end{array}$ & $9 \pm 2$ & $3.360 \pm 0.005$ & $0.103 \pm 0.005$ & 0 \\
\hline
\end{tabular}

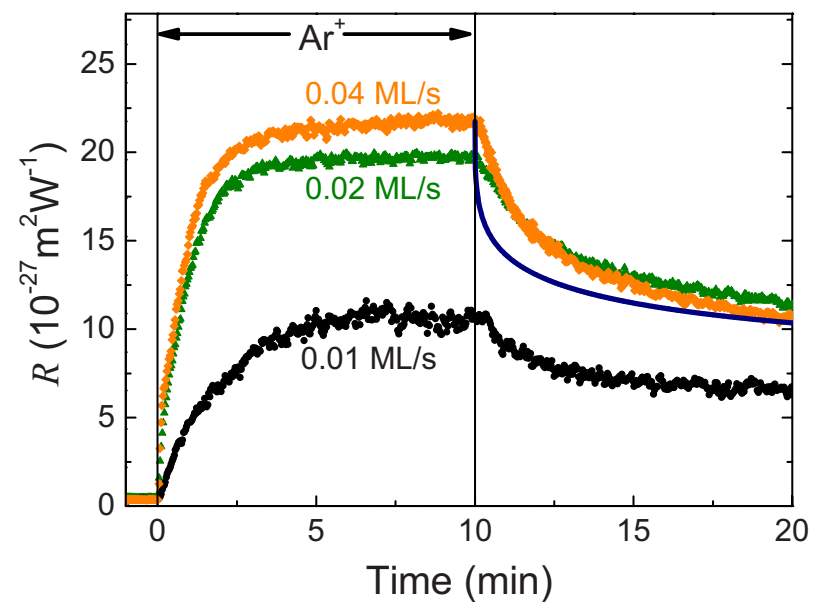

FIG. 6. (Color online) Time dependent SHG reflection coefficient for three $\mathrm{H}-\mathrm{Si}(100)$ samples before, during, and after $70 \mathrm{eV} \mathrm{Ar}^{+}$-ion bombardment, at ion fluxes of $0.01 \mathrm{ML} / \mathrm{s}, 0.02 \mathrm{ML} / \mathrm{s}$, and $0.04 \mathrm{ML} / \mathrm{s}$. The data were obtained at a photon energy of $\hbar \omega=1.0 \mathrm{eV}$. The solid line is described in the text and follows from a stretched exponential model.

used. After the ion bombardment was stopped the signal showed a considerable decay for several minutes. The final steady-state level of the response reached after the decay was clearly different from the starting condition, as expected. Remarkably, for the $0.04 \mathrm{ML} / \mathrm{s}$ experiment the increase from the initial $\mathrm{H}-\mathrm{Si}$ condition to the steady state during $\mathrm{Ar}^{+}$-ion bombardment is a factor 2 larger than expected from the spectra, which is not yet understood. The decrease afterward seems to be similar in magnitude to the change in the spectra when switching from $\mathrm{Ar}^{+}$-ion bombardment to $\mathrm{XeF}_{2}$ dosing.

Under the common assumption that the SHG signal is related to the surface density ${ }^{40}$ of a (defect) state and considering that the linear optical properties of the $a$-Si top layer do not depend on the ion flux, several conclusions can be drawn. The fact that the steady-state SHG signal depends on the ion flux and that the signal changes after termination of the bombardment indicates that the density of the states is not only governed by creation, but that there is also a process involved that reduces the density of these states during and after ion bombardment. Such a process could be the reconstruction of the silicon, or the passivation of the surface by background species in the vacuum setup. Because the difference between the 0.02 and $0.04 \mathrm{ML} / \mathrm{s}$ experiment is substantially smaller than the difference between the 0.01 and 0.02 $\mathrm{ML} / \mathrm{s}$ experiment, especially considering the quadratic behavior of the SHG intensity in the material property $\chi^{(2)}[\mathrm{cf}$. Eq. (2)], the surface density seems to reach a saturation value.

The initial slope in a figure of a time-resolved signal of a linear system is indicative of the fastest time constant involved. Investigation of the initial slope, instead of using the full signal, is especially valuable when the signal consists of multiple terms because the additional slower terms can substantially alter the shape of the full signal. Under the simple assumption that the contribution to the SHG intensity by the fastest term is quadratic in the density of some SHG generating electronic state (i.e., $\chi^{(2)}$ is assumed linear in this den- 
sity), the appropriate time scale can be obtained. The initial slopes deduced from the square root of the data in Fig. 6 scale as 1:6:12, where the fluxes scale as 1:2:4. For a SHG signal that is created by the $\mathrm{Ar}^{+}$-ion bombardment, one would expect that the initial slope scales linearly in the $\mathrm{Ar}^{+}$-ion flux. These numbers seem to agree with that picture and might only indicate an overestimation of the lowest flux. However, a note should be added here because such an approach is nontrivial in the nonlinear system of SHG. Equation (3) shows that the total signal generally consists of several contributions, which can effectively have any phase difference, due to linear propagation and the terms related to $\varphi_{q}$, and $\Gamma_{q}$. As a result, it is possible for the fastest term directly related to the $\mathrm{Ar}^{+}$-ion bombardment to increase, e.g., like $\chi_{L}^{(2)}(t) \sim[1-\exp (-t / \tau)]$, while the amplitude of the summed term in Eq. (2) is hardly affected initially, or even decreases.

For the decay of the SHG response after termination of the $\mathrm{Ar}^{+}$-ion bombardment, several explanations are proposed. The first explanation is that a thickness change due to bulk relaxation is responsible for the change in the signal, having an indirect influence on the SHG intensity because SHG is surface and interface specific. However, the change in layer thickness described by Eq. (4), using the values from Stevens et al. ${ }^{23}$ is too small to explain the changes in the signal. As a second idea, the bulk relaxation can mediate changes at the surface or interface and Eq. (4) can be assumed to describe the relevant $\chi_{L}^{(2)}$ term in Eq. (2). Using the values found by Stevens et al. to describe the time dependent behavior of one of the contributions and the values from Table I for the steady-state starting condition, the decay of the SHG signal is not described very well, however. The measured SHG signal decays slower than expected based on this explanation. Even adding a third (constant) contribution in Eq. (2) does not yield a reasonable fit (see Fig. 6). The third explanation is that the flux of background gas species in the setup, especially $\mathrm{H}_{2} \mathrm{O}$ and $\mathrm{O}_{2}$, interacts with the surface. Using kinetic theory the flux is calculated from the background pressure of $\sim 10^{-8} \mathrm{mbar}$ to be $0.05 \mathrm{ML} / \mathrm{s}$, based on the worst case scenario that the background pressure is fully dominated by $\mathrm{H}_{2} \mathrm{O}$ and $\mathrm{O}_{2} \cdot{ }^{9}$ In this way, $\mathrm{H}_{2} \mathrm{O}$ and $\mathrm{O}_{2}$ could quench the surface contribution in essentially the same way as $\mathrm{XeF}_{2}$ did. The time constant associated with such a flux, i.e., the time for each surface site to be exposed to an impinging molecule, is of the same order of magnitude as the decay of the SHG signal, making this explanation probable, but which needs to be confirmed by in situ surface analysis. Most likely the real behavior is described by a combination of these effects.

Besides varying the ion flux, information can also be obtained by varying the ion energy. Because higher energy ions create more damage per ion than low energy ions, resulting in a thicker $a$-Si layer, differences in the optical path length could already lead to differences in the SHG response. Figure 7 shows the time dependent evolution of the SHG signal for three different ion energies, i.e., 70, 200, and $1000 \mathrm{eV}$, at approximately the same $\mathrm{Ar}^{+}$-ion flux of $0.04 \mathrm{ML} / \mathrm{s}$, measured

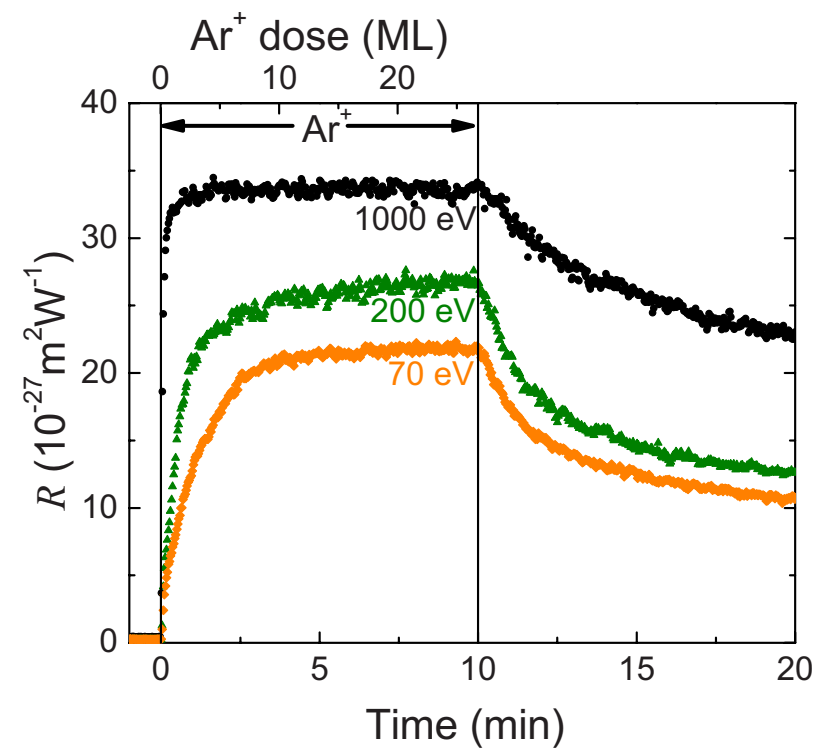

FIG. 7. (Color online) Time dependent SHG reflection coefficient for three $\mathrm{H}-\mathrm{Si}(100)$ samples before, during, and after $\mathrm{Ar}^{+}$-ion bombardment at an ion flux of $0.04 \mathrm{ML} / \mathrm{s}$. The ion energies were $70 \mathrm{eV}, 200 \mathrm{eV}$, and $1000 \mathrm{eV}$, respectively. The data were obtained at a fundamental photon energy of 1.0 $\mathrm{eV}$.

at $\hbar \omega=1.0 \mathrm{eV}$. Again, just like in the case of varying $\mathrm{Ar}^{+}$-ion fluxes, the behavior of the signal differs in three ways. First the initial increase rate is higher for higher ion energy. Second, the steady-state value increases with higher energy. Third, the decay after termination of the ion bombardment varies. The differences will be discussed separately.

The change in the initial increase with $\mathrm{Ar}^{+}$-ion energy is expected in the sense that with increasing ion energy, each ion creates more damage and, consequently, at the same flux, the $a$-Si layer is built up faster. Under the same assumptions as discussed before, the initial slopes can be extracted and they scale as $1: 2.5: 26$. When these numbers are compared with the ratios between the ion energies, i.e., 1:2.8:14, especially the latter value for an $\mathrm{Ar}^{+}$-ion energy of $1000 \mathrm{eV}$ seems high.

The dependency of the steady-state value on the ion energy can be explained in several ways. First, the SHG could, in principle, result from bulk contributions. However, Gielis et $a l^{9}$ showed that for the photon energy range of $\hbar \omega$ $=1.33-1.75 \mathrm{eV}$, the SHG signal was not related to the bulk, making it unlikely here as well. A second explanation could be that the change in the optical paths results in the difference. However, using the optical model discussed in Sec. I with the parameters in Table I, the different path lengths only yield a small change that shows the opposite effect. The third explanation is based on the fact that it is plausible that the density of states contributing to SHG increases with increasing ion energy. This could lead to an additional contribution in the SHG spectrum at higher ion energies, a contribution that was not yet apparent for the spectrum obtained for $70 \mathrm{eV}$ $\mathrm{Ar}^{+}$ions and therefore not necessary to be included in the fit 
of the spectrum. With the current data set, with limited SHG spectral data obtained for $1000 \mathrm{eV} \mathrm{Ar}{ }^{+}$ions, it is not possible to address this issue in more detail.

The decay of the SHG signal after termination of the ion bombardment varies with $\mathrm{Ar}^{+}$-ion energy without showing an unambiguous trend. The $1000 \mathrm{eV}$ ion energy experiment appears to decay slower than the other energy experiments. This behavior can be the result of the difference in the linear propagation due to different layer thicknesses, resulting in a different phase relation between the surface and interface terms. However, we are not yet able to adequately describe the decay consistently over all the samples.

\section{FINAL REMARKS}

We performed spectroscopic and time-resolved optical SHG during ion bombardment of $\mathrm{Si}(100)$ by $\mathrm{Ar}^{+}$ions with fluxes of 0.01, 0.02, and $0.04 \mathrm{ML} / \mathrm{s}$ and energies of 70, 200, and $1000 \mathrm{eV}$. We extended the previously reported photon energy range of $\hbar \omega=1.33-1.75 \mathrm{eV}$ downward by investigating the SHG signal over the photon energy range of $\hbar \omega$ $=0.76-1.14 \mathrm{eV}$. The behavior of the $a-\mathrm{Si} / c-\mathrm{Si}$ system at the lower photon energies is in agreement with the earlier observations at the higher photon energy range and can be explained by two-photon contributions located near $2 \hbar \omega$ $=3.4 \mathrm{eV}$ from both the surface and the $a-\mathrm{Si} / c-\mathrm{Si}$ interface . As discussed, the $2 \hbar \omega=3.4 \mathrm{eV}$ contributions are assigned to strained $\mathrm{Si}-\mathrm{Si}$ bonds at the surface and at the $a-\mathrm{Si} / c-\mathrm{Si}$ interface. The results in this study show no distinguishable sign of SHG activity by dangling bonds, which, in principle, can also contribute to the signal at this photon energy.

The SHG intensity shows an increase upon $\mathrm{Ar}^{+}$-ion bombardment, involving multiple time scales. The longer time scale agrees well with those observed in SE measurements and MD simulations, indicating that the changes in the signal are related to the processes investigated in those studies, i.e., to the buildup of the $a$-Si top layer due to the amorphization process. The fastest process observed in the time-resolved spectra in this work, i.e., the changes in the first seconds, were not observed previously and might be more specifically related to the SHG experiments and its high sensitivity to surface and interface processes.

Although the data presented in this article improve the understanding of the $\mathrm{Ar}^{+}$-ion sputtering of $\mathrm{Si}$, it is not yet possible to fully interpret the observed spectra and real-time data in terms of clear physical or chemical mechanisms. Combining the SHG measurements with other in situ techniques will help in unraveling those mechanisms. Furthermore, the parameters in the model describing the spectra can be resolved better by measuring, not only the amplitude, but also the relative phase of the SHG signal. ${ }^{41,42}$ This basically doubles the amount of information and results in less ambiguous fit parameters by decoupling the fit for the amplitude and phase of the SHG contributions. Such extended measurements will be pursued in our future work. Better spectral model parameters will also aid the modeling of the dynamic, time dependent behavior during sputtering, and could thus provide new information on the sputtering process.

\section{ACKNOWLEDGMENTS}

The authors would like to thank H. M. M. de Jong, R. F. Rumphorst, M. J. F. van de Sande, and J. J. A. Zeebregts for their skillful technical assistance.

\section{APPENDIX: REFLECTION COEFFICIENT}

To directly compare the spectra obtained in this work with the work from Gielis et al., ${ }^{9}$ who used photon counting, the SHG responses of the different experiments need to be expressed on a common scale. It is convenient to express the SHG response in terms of a reflection coefficient $R$, defined by $^{37}$

$$
R(2 \omega) \equiv \frac{I(2 \omega)}{\left[I_{\mathrm{in}}(\omega)\right]^{2}}
$$

because this coefficient is independent of the laser properties. In a simple approximation, the intensity $I$ of the laser beam is given by ${ }^{43,44}$

$$
I(\omega)=\frac{P_{\mathrm{av}}(\omega)}{t_{p} R_{\mathrm{rep}} A},
$$

where $t_{p}$ is the pulse duration, $R_{\text {rep }}$ is the repetition rate of the laser, and $A$ is the beam area. Together with the fact that the average power of the SHG beam is given by $P_{\mathrm{av}}(2 \omega)$ $=2 \hbar \omega S^{2 \omega}(2 \omega)$, where $S^{2 \omega}$ is the amount of photons radiated per second, and assuming that $t_{p}, R_{\text {rep }}$, and $A$ are the same for both the incoming and radiated light, Eq. (A1) can be written as

$$
R(2 \omega)=\frac{P_{\mathrm{av}}(2 \omega) t_{p} R_{\mathrm{rep}} A}{\left[P_{\mathrm{av}}(\omega)\right]^{2}}=2 \hbar \omega S^{2 \omega}(2 \omega) \frac{t_{p} R_{\mathrm{rep}} A}{\left[P_{\mathrm{av}}(\omega)\right]^{2}} .
$$

Because not all radiated SHG photons are detected, the measured signal $S$ needs to be corrected for the optical response $\mathbb{R}$ of the detection setup, i.e., $S(2 \omega)=\mathbb{R}(2 \omega) S^{2 \omega}(2 \omega)$. The optical response is obtained by multiplying the optical transmission of the colored filters and the polarizer in the exit beam path and the quantum efficiency of the PMT. ${ }^{9}$ Now Eq. (A3) can be written in experimental parameters as

$$
R(2 \omega)=\frac{2 \hbar \omega S(2 \omega)}{R(2 \omega)} \frac{t_{p} R_{\mathrm{rep}} A}{\left[P_{\mathrm{av}}(\omega)\right]^{2}} .
$$

The approach discussed so far is valid for the photon energy range of $\hbar \omega=1.33-1.75 \mathrm{eV}$. However, because of the high peak intensity in the fundamental beam, it is not possible to apply photon counting in the photon energy range of $\hbar \omega$ $=0.76-1.14 \mathrm{eV}$. Instead, the measured signal in our particular experiment results from multiple photons per laser pulse. Within a limited regime, the electrical charge generated in the PMT from a pulse of photons is linear in the amount of photons; therefore, the number of radiated photons per second can be calculated by considering the electronic circuit and the optical response. This is expressed in the following equation: 


$$
S^{2 \omega}(2 \omega)=\frac{S(2 \omega)}{\mathrm{R}(2 \omega)}=\frac{V C}{e G \mathbb{R}(2 \omega)} R_{\mathrm{rep}},
$$

where $V$ is the measured voltage, $C=30 \mathrm{pF}$ is the capacity used to store the photon charge of the PMT, $e$ is the electron charge, and $G$ is the gain of the PMT.

Because the gain of the PMT is not very accurately known, and the assumption that the beam parameters of the different lasers are the same is rather crude, it is expected that comparing the spectra is not perfect in absolute sense. Because these parameters only result in a relative difference between the two regimes, and the parameters of the laser in the $\hbar \omega=1.33-1.75 \mathrm{eV}$ photon energy range are better known, the spectra in the photon energy range of $\hbar \omega$ $=0.76-1.14 \mathrm{eV}$ are finally adjusted with a constant factor of 4.8. This factor results from fitting such that the SHG signal of the $\mathrm{H}-\mathrm{Si}$ sample in the photon energy range of $\hbar \omega$ $=0.76-1.14 \mathrm{eV}$ lies on single resonance fit of the spectrum in the $\hbar \omega=1.33-1.75 \mathrm{eV}$ photon energy range, which does not suffer from the ambiguities resulting from multiple contributions to the SHG signal. It is therefore considered a good reference for relating the reflection coefficients obtained for the two spectral ranges.

${ }^{1}$ International Technology Roadmap for Semiconductors, 2008 update, available at http://www.itrs.net.

${ }^{2}$ T. Lill and O. Joubert, Science 319, 1050 (2008).

${ }^{3}$ T. F. Heinz, M. M. T. Loy, and W. A. Thompson, J. Vac. Sci. Technol. B 3, 1467 (1985).

${ }^{4}$ U. Höfer, Appl. Phys. A: Mater. Sci. Process. 63, 533 (1996).

${ }^{5}$ T. Suzuki, Phys. Rev. B 61, R5117 (2000).

${ }^{6}$ W. Daum, H.-J. Krause, U. Reichel, and H. Ibach, Phys. Rev. Lett. 71, 1234 (1993).

${ }^{7}$ W. Daum, Appl. Phys. A: Mater. Sci. Process. 87, 451 (2007).

${ }^{8}$ G. Lüpke, Surf. Sci. Rep. 35, 75 (1999).

${ }^{9}$ J. J. H. Gielis, P. M. Gevers, A. A. E. Stevens, H. C. W. Beijerinck, M. C. M. van de Sanden, and W. M. M. Kessels, Phys. Rev. B 74, 165311 (2006).

${ }^{10}$ I. M. P. Aarts, J. J. H. Gielis, M. C. M. van de Sanden, and W. M. M. Kessels, Phys. Rev. B 73, 045327 (2006).

${ }^{11}$ J. J. H. Gielis, P. J. van den Oever, B. Hoex, M. C. M. van de Sanden, and W. M. M. Kessels, Phys. Rev. B 77, 205329 (2008).

${ }^{12}$ G. Erley and W. Daum, Phys. Rev. B 58, R1734 (1998).

${ }^{13}$ J. Ebothé, K. J. Plucinski, K. Nouneh, P. Roca i Cabarrocas, and I. V. Kityk, J. Nanomater. 2006, 63608 (2006).

${ }^{14}$ S. Alexandrova, P. Danesh, and I. A. Maslyanitsyn, Vacuum 69, 391 (2002).
${ }^{15}$ S. Lettieri, F. Merola, P. Maddalena, C. Ricciardi, and F. Giorgis, Appl. Phys. Lett. 90, 021919 (2007).

${ }^{16}$ S. Haraichi, F. Sasaki, S. Kobayashi, M. Komuro, and T. Tani, J. Vac. Sci. Technol. A 13, 745 (1995).

${ }^{17}$ S. Haraichi and F. Sasaki, J. Vac. Sci. Technol. A 15, 871 (1997).

${ }^{18}$ S. Haraichi and F. Sasaki, J. Vac. Sci. Technol. A 16, 3029 (1998).

${ }^{19}$ This equation holds for a monochromatic plane wave with $\mathbf{E}(\omega)$ the Fourier component of the electric field, such that $\mathbf{E}(\mathbf{r}, t)=\mathbf{E}(\omega) \exp [\imath(\mathbf{k} \cdot \mathbf{r}$ $-\omega t)]+$ c.c., with $\mathbf{k}$ the wave vector.

${ }^{20}$ G. Erley, R. Butz, and W. Daum, Phys. Rev. B 59, 2915 (1999).

${ }^{21}$ P. Lautenschlager, M. Garriga, L. Viña, and M. Cardona, Phys. Rev. B 36, 4821 (1987).

${ }^{22}$ A. Rumpel, B. Manschwetus, G. Lilienkamp, H. Schmidt, and W. Daum, Phys. Rev. B 74, 081303 (2006).

${ }^{23}$ A. A. E. Stevens, W. M. M. Kessels, M. C. M. van de Sanden, and H. C. W. Beijerinck, J. Vac. Sci. Technol. A 24, 1933 (2006).

${ }^{24}$ H. F. Winters and J. W. Coburn, Surf. Sci. Rep. 14, 162 (1992).

${ }^{25}$ G. Hobler and G. Otto, Mater. Sci. Semicond. Process. 6, 1 (2003).

${ }^{26}$ M. Fried, T. Lohner, W. A. M. Aarnink, L. J. Hanekamp, and A. van Silfhout, J. Appl. Phys. 71, 5260 (1992).

${ }^{27}$ J. Jellison and F. A. Modine, Appl. Phys. Lett. 69, 371 (1996).

${ }^{28}$ J. Ludwig, J. Eddy, O. Malis, and R. L. Headrick, Appl. Phys. Lett. 81, 2770 (2002).

${ }^{29}$ One monolayer (ML) is defined as the areal density of $\mathrm{Si}$ atoms on the $\mathrm{Si}(100)$ surface, i.e., $1 \mathrm{ML}=6.9 \times 10^{14} \mathrm{~cm}^{-2}$.

${ }^{30}$ J. Kakalios, R. A. Street, and W. B. Jackson, Phys. Rev. Lett. 59, 1037 (1987).

${ }^{31}$ R. S. Crandall, Phys. Rev. B 43, 4057 (1991).

${ }^{32}$ D. Humbird, D. B. Graves, A. A. E. Stevens, and W. M. M. Kessels, J. Vac. Sci. Technol. A 25, 1529 (2007).

${ }^{33}$ M. J. M. Vugts, G. J. P. Joosten, A. Vanoosterum, H. A. J. Senhorst, and H. C. W. Beijerinck, J. Vac. Sci. Technol. A 12, 2999 (1994).

${ }^{34}$ A. A. E. Stevens and H. C. W. Beijerinck, J. Vac. Sci. Technol. A 23, 126 (2005).

${ }^{35}$ P. M. Gevers, H. C. W. Beijerinck, M. C. M. van de Sanden, and W. M. M. Kessels, J. Appl. Phys. 103, 083304 (2008).

${ }^{36}$ The flux is defined relative to the surface of the sample, while in earlier work it was defined in the plane perpendicular to the ion beam.

${ }^{37}$ B. Koopmans, "Interface and bulk contributions in optical secondharmonic generation," Ph.D. thesis, Rijksuniversiteit Groningen, 1993.

${ }^{38}$ S. Bergfeld, B. Braunschweig, and W. Daum, Phys. Rev. Lett. 93, 097402 (2004).

${ }^{39}$ There appeared to be a small error in the software used by Gielis et al.

${ }^{40}$ In this context we mean the total areal density, not necessarily the one of the top surface.

${ }^{41}$ K. J. Veenstra, A. V. Petukhov, A. P. de Boer, and T. Rasing, Phys. Rev. B 58, R16020 (1998).

${ }^{42}$ P. T. Wilson, Y. Jiang, O. A. Aktsipetrov, E. D. Mishina, and M. C. Downer, Opt. Lett. 24, 496 (1999).

${ }^{43}$ G. Reider, M. Cernusca, and M. Hofer, Appl. Phys. B: Lasers Opt. 68, 343 (1999).

${ }^{44}$ J. J. H. Gielis, P. M. Gevers, I. M. P. Aarts, M. C. M. van de Sanden, and W. M. M. Kessels, J. Vac. Sci. Technol. A 26, 1519 (2008). 